\title{
Interfaces in Two-Dimensional Heterostructures of Transition Metal Dichalcogenides
}

Wu Zhou ${ }^{1}$, Junhao Lin ${ }^{2,1}$, Yongji Gong ${ }^{3,4}$, Xingli Wang ${ }^{5}$, Beng Kang Tay ${ }^{5}$, Jun Lou ${ }^{4}$, Zheng Liu $^{5}$, Sokrates T. Pantelides ${ }^{2,1}$, Pulickel M. Ajayan ${ }^{3,4}$

1. Materials Science \& Technology Division, Oak Ridge National Laboratory, Oak Ridge, TN 37831, USA

2. Department of Physics and Astronomy, Vanderbilt University, Nashville, TN 37235, USA

3. Department of Chemistry, Rice University, Houston, TX 77005, USA

4. Department of Materials Science \& NanoEngineering, Rice University, Houston, TX 77005, USA

5. School of Materials Science and Engineering, School of Electrical and Electronic Engineering Nanyang Technological University, 639798, Singapore

Two-dimensional (2D) transition-metal dichalcogenides (TMDs) are promising candidates for flexible nanoelectronics, with exceptional optical and electrical properties at monolayer thickness. Monolayers of different TMDs can be further combined to create van der Waals heterostructures, where multiple 2D layers are stacked vertically layer-by-layer, or stitched seamlessly in plane to form lateral heterojunctions. The coupling between the different 2D components provides unique opportunities for bandgap engineering and can create very unusual properties at the interface [1-4]. Revealing the atomic structure, including the stacking orientation, stacking order, and chemical inter-diffusion, is therefore important for understanding the novel properties generated by the heterostructure interfaces.

Recently, we have demonstrated a simple one-step vapor phase growth of high quality heterostructures of $\mathrm{WS}_{2}$ and $\mathrm{MoS}_{2}$ [1]. High temperature growth yields predominantly vertically stacked bilayer heterostructures, while low temperature growth creates mostly lateral heterostructures of $\mathrm{WS}_{2}$ and $\mathrm{MoS}_{2}$ within the same monolayer. The atomic structure and electronic properties of the heterostructure interfaces are studied by aberration-corrected scanning transmission electron microscopy (STEM) annular dark field (ADF) imaging, electron energy-loss spectroscopy (EELS) at low voltage, and density functional calculations,

STEM-ADF imaging reveals that the vertical heterostructures were obtained with $\mathrm{WS}_{2}$ epitaxially grown on top of the $\mathrm{MoS}_{2}$ monolayer, following the preferred 2H stacking (Figure 1). A small amount ( 3\%) of W substitution in the $\mathrm{MoS}_{2}$ layer and Mo substitution in the $\mathrm{WS}_{2}$ layer was observed in the sample, which should only have minimum effect on the properties of the $\mathrm{MoS}_{2}$ and $\mathrm{WS}_{2}$ monolayers at such low concentration. Photoluminescence (PL) analysis shows that the $\mathrm{MoS}_{2}$ and $\mathrm{WS}_{2}$ layers in the bilayer heterostructure, on one hand, behave as individual monolayers, and, on the other hand, generate a new direct band gap of $\mathrm{WS}_{2} / \mathrm{MoS}_{2}$ heterostructure via interlayer coupling owing to the clean interface.

Atomically sharp interfaces were frequently observed in the lateral heterojunctions, with seamless connection and abrupt transition between the $\mathrm{MoS}_{2}$ and $\mathrm{WS}_{2}$ lattice within a single atomic row. Most of the abrupt lateral interfaces were achieved by lateral epitaxial growth of $\mathrm{WS}_{2}$ on fresh $\mathrm{MoS}_{2}$ edges along the zigzag direction, and sharp armchair interfaces were only occasionally observed. Lateral interfaces with large chemical inter-diffusion over a width of a 
few hundred nanometers were also observed, presumably due to local fluctuations in the growth conditions. The different degrees of chemical inter-diffusion are most likely responsible for the observed inhomogeneous PL enhancement along the lateral interfaces.

Besides the $\mathrm{WS}_{2}-\mathrm{MoS}_{2}$ system, results from WSe $/ \mathrm{MoSe}_{2}$ heterostructures will also be discussed, which provides insights into the growth mechanism and guidance for the growth of superlattice structures [5].

\section{References:}

[1] Y Gong et al, Nature Materials 13 (2014), P. 1135-1142.

[2] C Huang et al, Nature Materials 13 (2014), P. 1096-1101.

[3] X Duan et al, Nature Nanotechnology 9 (2014), P. 1024-1030.

[4] AK Geim \& IV Grigorieva, Nature 499 (2013) P. 419-425.

[5] This research was supported in part a Wigner Fellowship of Oak Ridge National Laboratory (WZ), by the Office of Science, Basic Energy Science, Materials Sciences and Engineering Division, U.S. DOE (WZ), by U.S. DOE grant DE-FG02-09ER46554 (JL, STP), and through a user project supported by ORNL's Center for Nanophase Materials Sciences (CNMS), which is sponsored by the Scientific User Facilities Division, Office of Basic Energy Sciences, U.S. DOE. YG and PMA were supported by Army Research Office MURI grant W911NF-11-1-0362.

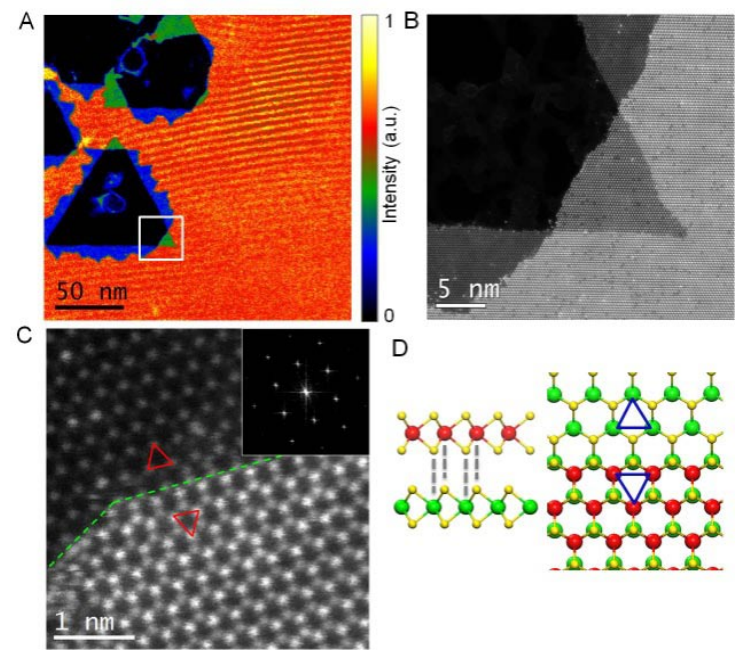

Figure 1. STEM-ADF imaging of vertical heterostructure of $\mathrm{WS}_{2} / \mathrm{MoS}_{2}$ at different magnifications. Figure A is shown in color scale where monolayer $\mathrm{MoS}_{2}$ is in blue, monolayer $\mathrm{WS}_{2}$ in green and $\mathrm{WS}_{2} / \mathrm{MoS}_{2}$ bilayer in orange. (D) is the structure model illustrating the $2 \mathrm{H}$ stacking [1].

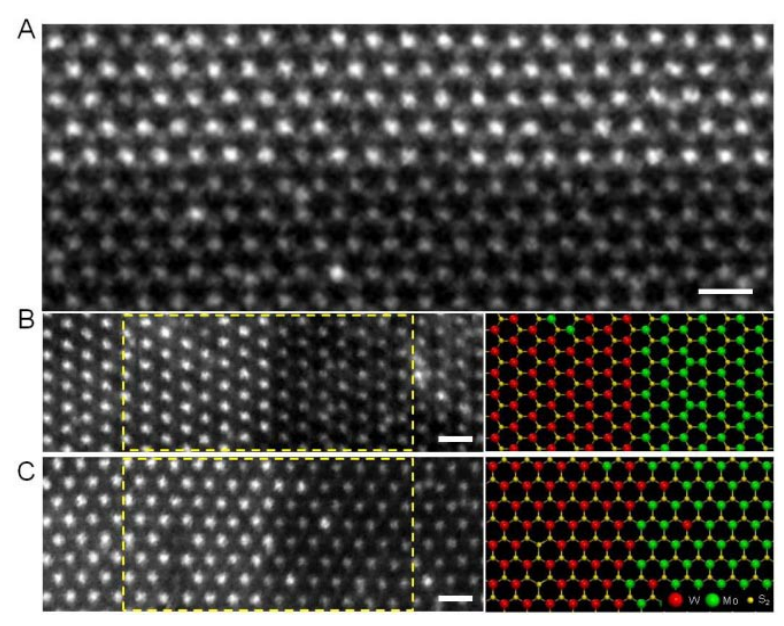

Figure 2. STEM-ADF imaging of atomically sharp lateral interfaces between $\mathrm{WS}_{2}$ and $\mathrm{MoS}_{2}$ along the zigzag (A, B) and armchair (C) directions. Scale bars: $0.5 \mathrm{~nm}$ [1]. 